\title{
Burkholderia cepacia, la evolución en una paciente con fibrosis quística hasta el desarrollo del síndrome cepacia
}

\author{
Burkholderia cepacia, clinical course in a cystic fibrosis patient \\ through the development of cepacia syndrome
}

\author{
Yolotl Hilario Sánchez-Carrillo,* Tania Romero-López, ${ }^{\ddagger}$ Cyntia Ibanes-Gutiérrez,* Napoleón González-Saldaña ${ }^{\S}$ \\ * Residente de Infectología Pediátrica. \\ ‡ Residente de Pediatría. \\ $\S$ Jefe del Servicio de Infectología Pediátrica. \\ Instituto Nacional de Pediatría.
}

RESUMEN

Burkholderia cepacia es un patógeno oportunista en los pacientes con fibrosis quística (FQ) cuyo impacto clínico se asocia con incremento de la morbilidad y mortalidad, llegando a causar el denominado síndrome cepacia. Presentamos el caso de una paciente de sexo femenino de cinco años, que a lo largo de su evolución presentó los diferentes espectros clínicos, desde la colonización por B. cepacia, hasta el deterioro progresivo de la función respiratoria y finalmente síndrome cepacia, que ocasionó su deceso. La edad de colonización y la subespecie aislada, en los pacientes con FQ impactan directamente en la supervivencia a largo plazo. B. cepacia puede adquirirse en el medio hospitalario o al estar en contacto con pacientes portadores. El síndrome cepacia se caracteriza por ser una complicación generalmente fatal. El tratamiento suele ser complejo debido a las resistencias del microorganismo, reportándose el uso de esquemas de hasta tres antimicrobianos, con resultados variables en los diferentes reportes.

Palabras clave: Fibrosis quística, complejo Burkholderia cepacia, síndrome cepacia, agentes antiinfecciosos.

\section{INTRODUCCIÓN}

Burkholderia cepacia, un bacilo móvil Gram negativo, de la familia Burkholderiaceae, género Burkholderia, fue descrito por primera vez en 1950 por Burkholder como un patógeno putrefactor de las cebollas. ${ }^{1} \mathrm{~A}$

Financiamiento: Ninguno.

Conflicto de intereses: Ninguno.

Rev Latin Infect Pediatr 2020; 33 (4): 210-214
ABSTRACT

Burkholderia cepacia is an opportunistic pathogen in patients with cystic fibrosis. Its clinical impact is associated with increased morbidity and mortality, causing the so-called cepacia syndrome. We present the case of a five year-old female, that throughout the history of her disease presented with different clinical scenarios within the spectrum of the syndrome, from colonization by $\mathrm{B}$. cepacia to progressive deterioration of pulmonary function and finally the cepacia syndrome, which caused her death. The age of colonization and the isolated subspecies, in cystic fibrosis patients, have a direct impact on long term survival. B. cepacia can be acquired in the hospital environment or through contact with carrier patients. Cepacia syndrome is characterized by generally being a fatal complication. The treatment is usually complex due to the microorganism's resistance, with reports of up to three antimicrobials, with variable results in the different series.

Keywords: Cystic fibrosis, Burkholderia cepacia complex, cepacia syndrome, anti-infective agents.

partir de 1980 fue identificado como un patógeno oportunista en los pacientes con fibrosis quística (FQ) y en 1984 se publicó por primera vez el impacto clínico que ocasiona en estos pacientes. ${ }^{2} \mathrm{La}$ infección pulmonar se asocia con incremento de la morbilidad y mortalidad, llegando a causar neumonía

Citar como: Sánchez-Carrillo YH, Romero-López T, Ibanes-Gutiérrez C, González-Saldaña N. Burkholderia cepacia, la evolución en una paciente con fibrosis quística hasta el desarrollo del síndrome cepacia. Rev Latin Infect Pediatr. 2020; 33 (4): 210-214. https://dx.doi.org/10.35366/96850 
Rev Latin Infect Pediatr 2020; 33 (4): 210-214

fulminante y septicemia, lo que se denomina síndrome cepacia. ${ }^{3}$

En los pacientes con FQ, la infección por B. cepacia se puede categorizar en tres espectros clínicos: ${ }^{2}$

1. Infección o colonización sin alteraciones de la función pulmonar.

2. Infección con disminución de la función pulmonar de manera acelerada.

3. Insuficiencia respiratoria aguda con bacteriemia, neumonía necrosante, leucocitosis y muerte al cabo de semanas o meses.

El primer aislamiento de $B$. cepacia en secreción respiratoria supone un mal pronóstico. ${ }^{1}$ Aproximadamente $20 \%$ de los pacientes infectados desarrollan síndrome cepacia. Se pensaba que el síndrome cepacia ocurría próximo al primer aislamiento, pero se han reportado casos hasta 31 meses después. ${ }^{4}$

\section{PRESENTACIÓN DEL CASO}

Presentamos el caso de una niña de cinco años, con diagnóstico de $F Q$ desde los seis meses de edad, con mutación heterocigota para $\Delta \mathrm{F} 508$, colonizada por Pseudomonas aeruginosa y Staphylococcus aureus desde los 21 meses. Durante su quinta exacerbación, a los dos años nueve meses, se aisló por primera vez Burkholderia cepacia en secreción respiratoria, a los cuatro años dos meses, en su octava exacerbación, cursó con septicemia por $B$. cepacia sin insuficiencia respiratoria; sin embargo, a partir de ese momento presentó deterioro acelerado de la función pulmonar.

A los cinco años ocho meses, ingresó por su decimocuarta exacerbación y, debido al contexto de la pandemia de COVID-19, se realizó PCR de SARS-CoV-2 que resultó negativa. Recibió tratamiento empírico inicial con ceftazidima, amikacina y vancomicina por considerarse colonizada por los microorganismos antes mencionados. Tuvo una evolución tórpida, por lo que requirió aporte de oxígeno por puntas de alto flujo; tras cuatro días de administración del esquema inicial, se reportó en cultivo de secreción respiratoria crecimiento de $S$. aureus meticilino resistente, se suspendió vancomicina y se inició linezolid para mejorar la disponibilidad en tejido pulmonar. Siete días después de su ingreso, se aisló en secreción respiratoria $B$. cepacia, por lo que se indicaron dos antibióticos de primera línea: ceftazidima y trimetoprima-sulfametoxazol (TMP-SMX) por vía sistémica, con tobramicina inhalada. Después de 11 días de hospitalización con una evolución tórpida, ya requiriendo apoyo con un dispositivo de presión positiva continua por mascarilla nasal, se reportó sensibilidad a meropenem y ceftazidima y resistencia a TMP-SMX y levofloxacino, por lo que se decidió descontinuar TMP-SMX e iniciar meropenem.

La evolución fue estacionaria durante los siete días posteriores al inicio de meropenem, tiempo en que presentó deterioro de la función respiratoria, requiriendo ventilación mecánica invasiva. Se aisló $B$. cepacia en hemocultivo y, debido al riesgo-beneficio, se agregó piperacilina-tazobactam al esquema de tratamiento. Asimismo, recibió inmunoglobulina intravenosa humana inespecífica.

Dentro de las complicaciones que presentó, padeció una infección asociada a catéter venoso central por $B$. cepacia, diagnosticada por criterio cualitativo de diferencia de tiempo entre la toma de hemocultivo central y periférico. Presentó igualmente una hemorragia intracraneal secundaria al estado crítico.

Después de 30 días de hospitalización, se logró el retiro de la ventilación mecánica invasiva y por la mejoría clínica se suspendió la ureidopenicilina; desafortunadamente, días después progresó de nuevo a insuficiencia respiratoria secundaria al daño pulmonar, lo cual se evidenció por radiografías, ya que el estado del caso no permitió realizar otros estudios funcionales (Figura 1). Por último, los padres con apoyo del servicio de soporte para la calidad de vida tomaron la decisión de que se limitaran los esfuerzos terapéuticos, dando medidas de confort y manejo del dolor hasta su fallecimiento.

\section{DISCUSIÓN}

La colonización por el complejo Burkholderia cepacia en pacientes con FQ disminuye la supervivencia a largo plazo, por lo tanto, la edad en que adquieren este microorganismo es crucial. En países desarrollados se reportan edades de colonización que van desde meses a años, Coutinho y colaboradores encontraron en centros de fibrosis quística en Portugal una edad promedio del primer aislamiento a los 10 años. ${ }^{5}$ Estudios sugieren que el mal pronóstico se asocia a la subespecie aislada (casos con $B$. cenocepacia padecen menor tiempo hasta el fallecimiento respecto a otras especies) y a la frecuencia de colonización en conjunto con otros microorganismos. ${ }^{6}$ En México y Latinoamérica, no existen estudios 

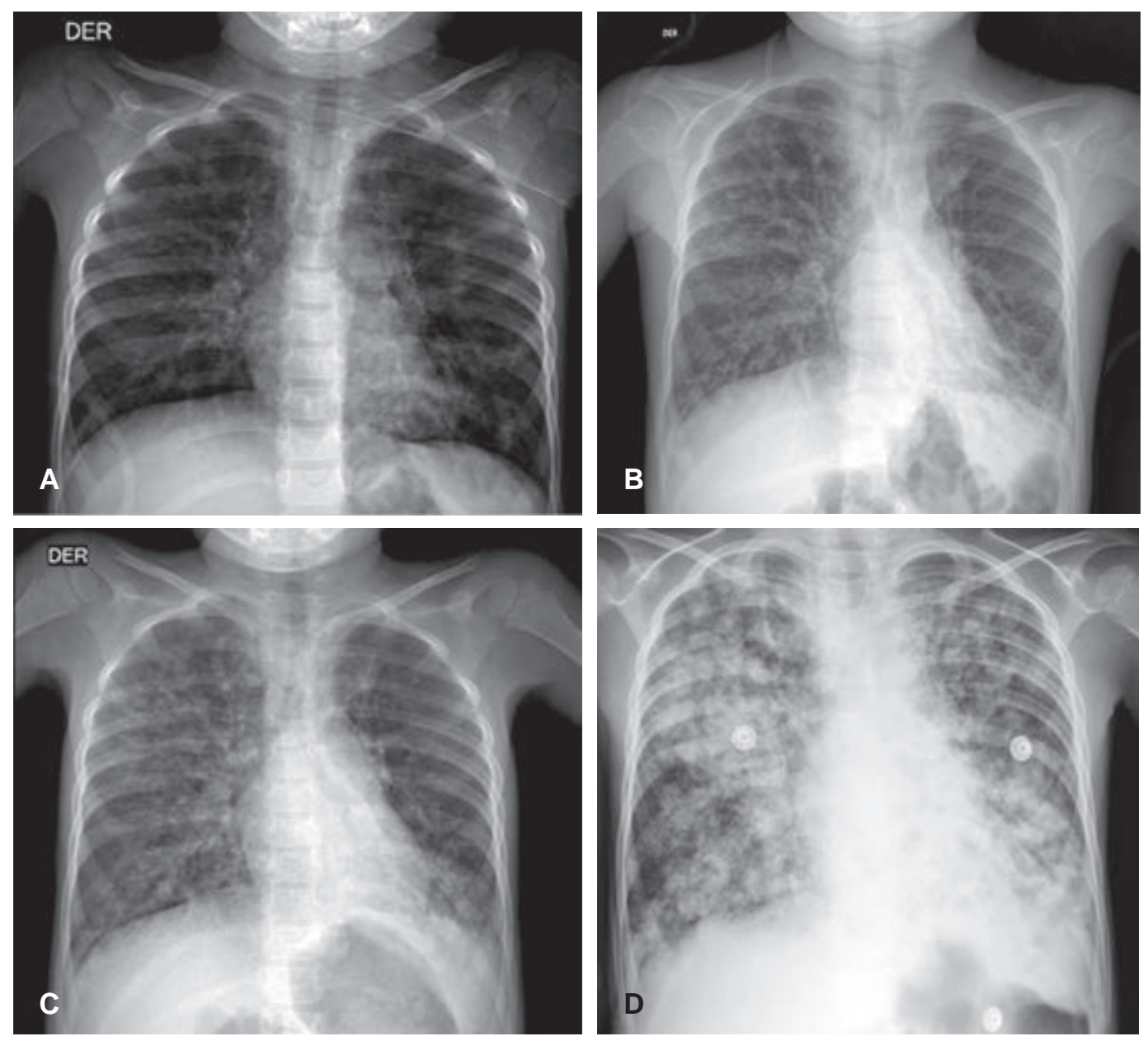

Figura 1: Evolución radiográfica. A) Radiografía al momento del primer aislamiento de B. cepacia. Se observan imágenes sugestivas de bronquiectasias parahiliares de predominio derecho, con horizontalización de arcos costales. B) Radiografía posterior a la bacteriemia sin insuficiencia respiratoria durante su octava exacerbación. Se aprecia un infiltrado bronquioalveolar de predominio en hemitórax derecho, con discreto aumento de las imágenes sugestivas de bronquiectasias. C) Radiografía al inicio de la decimocuarta exacerbación, donde se aprecia fibrosis en las bases pulmonares, con un infiltrado intersticial bilateral, además de progresión de las dilataciones bronquiales, sobre todo en hilio izquierdo. D) Radiografía de tórax en la fase terminal de la paciente, donde es evidente el daño pulmonar, con un infiltrado algodonoso que es sugestivo de hemorragia pulmonar.

específicos que otorguen información acerca de la edad de colonización por este microorganismo, la edad temprana de adquisición en nuestra paciente fue un hecho desfavorable.

El síndrome cepacia se caracteriza por ser una complicación generalmente fatal en los pacientes con $\mathrm{FQ}^{7}$ y $B$. cepacia, además de diseminarse en el medio hospitalario, se ha reportado capaz de transmitirse de manera extrahospitalaria al estar en contacto con pacientes portadores, ${ }^{8,9}$ por ello deberían tenerse precauciones acerca del manejo de los pacientes colonizados.
El tratamiento suele ser complejo debido a que la gran mayoría de las cepas de $B$. cepacia son resistentes a múltiples antibióticos, ${ }^{10}$ dos revisiones sistemáticas en 2019 no lograron encontrar evidencia para generar información útil sobre el manejo de estos pacientes y hacen evidente la necesidad de diseñar estudios con este objetivo. ${ }^{11,12}$ Se reporta el uso de esquemas basados en uno, dos o hasta tres antimicrobianos, con resultados variables. ${ }^{13}$ Las opciones terapéuticas incluidas en el CLSI 2020 son ticarcilina-clavulanato, ceftazidima, meropenem, minociclina, levofloxacino, trimetoprima-sulfametoxa- 
Rev Latin Infect Pediatr 2020; 33 (4): 210-214

zol y algunos medicamentos con posible resistencia inducida por mutaciones en algunas cepas, que incluye piperacilina-tazobactam. ${ }^{14}$ De acuerdo a los recursos del hospital y sensibilidades reportadas, adaptamos el mejor esquema para nuestro caso, teniendo en mente que la evolución de los pacientes colonizados por B. cepacia es impredecible y dependiente de varios factores: por un lado, la habilidad del microorganismo para sobrevivir en el parénquima pulmonar de los sujetos con FQ, donde los antibióticos tienen una penetración variable, por otro lado, la posibilidad de invadir el torrente sanguíneo. Todo lo anterior podría contribuir a una disociación entre las susceptibilidades in vitro y en la clínica. Por último, hay que tener en cuenta la capacidad del microorganismo de detonar una respuesta inmune exagerada, pero inefectiva. ${ }^{13}$ Aunque logramos obtener hemocultivos negativos tras el manejo con meropenem, trimetoprima-sulfametoxazol, piperacilina-tazobactam intravenosos y tobramicina inhalada, el daño pulmonar fue tan grave que condujo a la paciente a la muerte.

Dada la dificultad para el manejo de los pacientes, existen reportes de tratamientos alternativos. Una unidad de fibrosis quística en Manchester ha implementado el tratamiento con metilprednisolona, inmunoglobulina y ciclosporina para mediar la respuesta inflamatoria. ${ }^{1}$ Gilchrist y colaboradores reportan el caso de un adulto con síndrome cepacia tratado con ciclosporina como mediador de la respuesta inflamatoria, obteniendo un resultado favorable con la supervivencia del paciente, ${ }^{15}$ y Kazachkov y su equipo reportan el caso de una paciente pediátrica que presentó síndrome cepacia, como parte del tratamiento se administró metilprednisolona a dosis de $4 \mathrm{mg}$ por kilogramo por día con respuesta favorable. ${ }^{16}$

Una vez que un microorganismo se encuentra en la circulación sistémica, es capaz de implantarse en diferentes sitios, lo que dificulta la erradicación. En nuestro caso, desarrolló una infección asociada a catéter, se retiró el dispositivo al tratarse de un bacilo Gram negativo multidrogorresistente, de acuerdo con las guías internacionales. ${ }^{17}$

\section{CONCLUSIÓN}

B. cepacia es un patógeno de importancia en los pacientes con $F Q$, pues incide directamente en la morbilidad y mortalidad, de ahí que se requieran medidas de control para prevenir o retrasar la colo- nización, más aún, que hasta este momento no se encuentran disponibles recomendaciones con alto nivel de evidencia acerca del manejo y tratamiento de cualquiera de los espectros clínicos que puede producir. Por ese motivo, se necesitan estudios que busquen definir y homogeneizar tratamientos más efectivos para este grupo de pacientes.

\section{REFERENCIAS}

1. Jones AM, Dodd ME, Webb AK. Burkholderia cepacia: current clinical issues, environmental controversies and ethical dilemmas. Eur Respir J. 2001; 17 (2): 295-301.

2. Zahariadis G, Levy MH, Burns JL. Cepacia-Like syndrome caused by Burkholderia mutivorans. Can J Infect Dis. 2003; 14 (2): 123-125.

3. Jones A, Dodd M, Govan J, Barcus V, Doherty CJ, Morris $\mathrm{J}$ et al. Burkholderia cenocepacia and Burkholderia multivorans: influence on survival in cystic fibrosis. Thorax. 2004; 59 (11): 948-951.

4. Blackburn L, Brownlee K, Conway S, Denton M. "Cepacia syndrome" with Burkholderia multivorans, 9 years after initial colonization. Journal of Cystic Fibrosis. 2004; 3 (2): 133134.

5. Coutinho CP, dos Santos SC, Madeira A, Mira NP, Moreira AS, Sá-Correia I. Long-term colonization of the cystic fibrosis lung by Burkholderia cepacia complex bacteria: epidemiology, clonal variation, and genome-wide expression alterations. Fron Cell Infect Microbiol. 2011; 1: 12.

6. Somayaji R, Yau Y, Tullis E, LiPuma JJ, Ratjen F, Waters V. Clinical outcomes associated with Burkholderia cepacia complex infection in patients with cystic fibrosis. Ann Am Thorac Soc. 2020; 10.1513/AnnalsATS.202003204OC.

7. Manno G, Dalmastri C, Tabacchioni S, Vandamme P, Lorini R, Minicucci L, Bevivino A. Epidemiology and clinical course of Burkholderia cepacia complex infections, particularly those caused by different Burkholderia cenocepacia strains, among patients attending an Italian cystic fibrosis center. J Clin Microbiol. 2004; 42 (4): 1491-1497.

8. Hindo H, Sigley C, Karlson K. Cepacia syndrome in an adolescent with cystic fibrosis. Infectious Diseases in Clinical Practice. 2008; 16 (3): 198-200.

9. Millar-Jones L, Ryley HC, Paull A, Goodchild MC. Transmission and prevalence of Burkholderia cepacia in welsh cystic fibrosis patients. Respiratory Medicine. 1998; 92 (2): 178183.

10. Aaron SD, Ferris W, Henry DA, Speert DP, MacDonald NE. Multiple combination bactericidal antibiotic testing for patients with cystic fibrosis infected with Burkholderia cepacia. Am J Respir Crit Care Med. 2000; 161 (4): 12061212.

11. Regan $\mathrm{KH}$, Bhatt J. Eradication therapy for Burkholderia cepacia complex in people with cystic fibrosis. Cochrane Database Syst Rev. 2019; 4: CD009876.

12. Lord R, Jones AM, Horsley A. Antibiotic treatment for Burkholderia cepacia complex in people with cystic fibrosis experiencing a pulmonary exacerbation. Cochrane Database Syst Rev. 2020; 4: CD009529.

13. Grimwood K, Kidd TJ, Tweed M. Successful treatment of cepacia syndrome. J Cyst Fibros. 2009; 8 (4): 291-293. 
Rev Latin Infect Pediatr 2020; 33 (4): 210-214

14. CLSI. Performance Standards for Antimicrobial Susceptibility Testing. 30th ed. CLSI Supplement M100. Wayne, PA: Clinical and Laboratory Standards Institute; 2020.

15. Gilchrist FJ, Webb AK, Bright-Thomas RJ, Jones AM. Successful treatment of cepacia syndrome with a combination of intravenous cyclosporin, antibiotics and oral corticosteroids. J Cyst Fibros. 2012; 11 (5): 458-460.

16. Kazachkov M, Lager J, LiPuma J, Barker PM. Survival following Burkholderia cepacia sepsis in a patient with cystic fibrosis treated with corticosteroids. Pediatr Pulmonol. 2001; 32 (4): 338-340.

17. Rupp ME, Karnatak R. Intravascular catheter-related bloodstream infections. Infect Dis Clin. 2018; 32 (4): 765787.

Correspondencia:

Dr. Yolotl Hilario Sánchez Carrillo

E-mail: yolotlhsc@gmail.com

Las instrucciones para los autores se encuentran disponibles para descarga en la página de Internet:

https://www.medigraphic.com/pdfs/infectologia/lip-instr.pdf

También puedes accesar por medio del código QR:

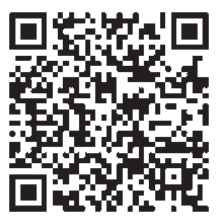

